\title{
Motor Cortex and Hand Motor Skills: Structural Compliance in the Human Brain
}

\author{
Katrin Amunts, ${ }^{1 *}$ Gottfried Schlaug, ${ }^{2 *}$ Lutz Jäncke, ${ }^{3}$ Helmuth Steinmetz, ${ }^{4}$ \\ Axel Schleicher, ${ }^{1}$ Andreas Dabringhaus, ${ }^{1}$ and Karl Zilles ${ }^{1}$ \\ ${ }^{1}$ C. E O. Vogt Brain Research Institute, Heinrich Heine University, Düsseldorf, Germany \\ ${ }^{2}$ Department of Neurology, Beth Israel Hospital and Harvard Medical School, Boston, Massachusetts \\ ${ }^{3}$ Institute of Medicine, Research Center Jülich, Jülich, Germany \\ ${ }^{4}$ Department of Neurology, Heinrich Heine University, Düsseldorf, Germany
}

\begin{abstract}
Recent studies in humans and nonhuman primates have shown that the functional organization of the human sensorimotor cortex changes following sensory stimulation or following the acquisition of motor skills. It is unknown whether functional plasticity in response to the acquisition of new motor skills and the continued performance of complicated bimanual movements for years is associated with structural changes in the organization of the motor cortex. Professional musicians, especially keyboard and string players, are a prototypical group for investigating these changes in the human brain. Using magnetic resonance images, we measured the length of the posterior wall of the precentral gyrus bordering the central sulcus (intrasulcal length of the precentral gyrus, ILPG) in horizontal sections through both hemispheres of right-handed keyboard players and of an age- and handedness-matched control group. Lacking a direct in vivo measurement of the primary motor cortex in humans, we assumed that the ILPG is a measure of the size of the primary motor cortex. Left-right asymmetry in the ILPG was analyzed and compared between both groups. Whereas controls exhibited a pronounced left-larger-than-right asymmetry, keyboard players had more symmetrical ILPG. The most pronounced differences in ILPG between keyboard players and controls were seen in the most dorsal part of the presumed cortical hand representation of both hemispheres. This was especially true in the nondominant right hemispheres. The size of the ILPG was negatively correlated with age of commencement of musical training in keyboard players, supporting our hypothesis that the human motor cortex can exhibit functionally induced and long-lasting structural adaptations. Hum. Brain Mapping 5:206-215, 1997 @ 1997 Wiley-Liss, Inc.
\end{abstract}

Key words: human motor cortex; asymmetry; plasticity; handedness; MRI; musicians; hand motor skill

\section{INTRODUCTION}

Contract Grant sponsor: Deutsche Forschungsgemeinschaft; Contract Grant number: SFB 199, A6.

${ }^{*}$ Correspondence to: Katrin Amunts, C. \& O. Vogt Brain Research Institute, Heinrich Heine University, Düsseldorf, Germany. E-mail: katrin@hirn.uni-duesseldorf.de or Gottfried Schlaug, Dept. of Neurology, Beth Israel Deaconess Medical Center, 330 Brookline Avenue, Boston, MA 02215, USA. E-mail: gschlaug@mercury.bidmc.harvard.edu Received for publication 22 January 1997; accepted 10 April 1997
Electrophysiological and neuroimaging studies in humans revealed adaptive changes in the cortical motor hand representations in response to learning and the acquirement of new motor skills [Jenkins et al., 1994; Pascual-Leone et al., 1993, 1995a,b; Schlaug et al., 1994; Karni et al., 1995]. Similar functional changes have been reported after stimulation of primary somatosensory and motor areas in nonhuman primates

(C) 1997 Wiley-Liss, Inc. 
and cats [Merzenich et al., 1988; Recanzone et al., 1990; Jacobs and Donoghue, 1991; Nudo et al., 1992; Wang et al., 1995]. Pascual-Leone et al. [1995a] showed that over the course of 5 days, as subjects learned a five-finger exercise on the piano, the cortical motor areas targeting the long finger flexor and extensor muscles enlarged. Others suggested that the early acquirement of fine sensorimotor skills leads to lasting functional changes, such as having a larger sensory finger representation in string players [Elbert et al., 1995]. The underlying neurophysiological changes are still a matter of intense research. Possible modifications could include a strengthening of existing synapses, the formation of new synapses, or recruitment of cortical tissue into the activated cortex that was previously not recruited [Donoghue and Sanes, 1988; Merzenich et al., 1988; Recanzone et al., 1990; Jacobs and Donoghue, 1991; Nudo et al., 1992; Pascual-Leone et al., 1993, 1995a,b; Karni et al., 1995; Jones et al., 1996; Kleim et al., 1996].

In a previous study, we found evidence for structural adaptations in the interhemispheric connectivity of frontal motor-related regions between musicians with a different commencement of musical training. The anterior corpus callosum was larger in musicians with an early commencement of musical training (before age 7) compared to musicians starting later (after age 7) and right-handed controls [Schlaug et al., 1995a]. Since this structural difference was seen in musicians with an early commencement of musical training, it was argued that this might represent a structural cerebral adaptation (e.g., thicker myelinated transcallosal fibers or a greater amount of transcallosal fibers) which have been triggered by a requirement for performing and continuously practicing complicated bimanual finger movements. These results indicated that the corpus callosum underwent an adaptation in the young brain which seemed to coincide with the late myelination of the corpus callosum [Yakovlev and Lecours, 1967]. Since late-beginning musicians (some of the musicians in this study were quite accomplished performers) did not show this anatomical difference and as a matter of fact did not differ significantly from a right-handed nonmusician control group, a selectional hypothesis (e.g., a larger anterior corpus callosum would endow a child to become skilled at playing a musical instrument at a young age) was deemed to be less likely to explain these anatomical differences. A selectional hypothesis would explain the structural differences in the brains of musicians compared with controls as an effect of a selection of people with, for example, larger corpora callosa inherent in the demands of musical training and competition.
Encouraged by our previous findings and by the results of Elbert et al. [1995] and Schlaug et al. [1995b], we wanted to investigate whether the early commencement and long duration of motor learning and practice of complex bimanual finger movements in musicians might lead to persistent, macrostructural adaptations within the motor cortex, which may underlie the representational plasticity observed in persons while acquiring and continuously performing fine motor skills [Pascual-Leone et al., 1993, 1995a,b; Schlaug et al., 1994].

There is also previous evidence that the primary motor cortex might show a structural correlate of handedness. Several groups [White et al., 1994; Foundas et al., 1995; Amunts et al., 1996] have shown that estimates of the primary motor cortex revealed a left-larger-than-right motor cortex size in right-handed subjects. While right-handers showed a left-larger-thanright motor cortex size (as indexed by the ILPG), left-handers showed a right-larger-than-left ILPG [Amunts et al., 1996]. These interhemispheric asymmetries were thought to represent structural correlates of handedness. A similar suggestion was made on the basis of results from a postmortem study on human brains with unknown handedness [White et al., 1994]. In roughly $2 / 3$ of these brains, the left central sulcus was deeper than the right. In this regard, it is also important that several studies have found evidence that handedness and distal hand motor skills are much more symmetrical in professional musicians [Christman, 1993; Schlaug et al., 1995a; Waldron and Anton, 1995].

On the basis of all these findings we hypothesized that the size of cortical motor areas could differ between musicians with a history of intense bimanual hand skill training and appropriate nonmusician controls. In this study, we attempted to answer the following questions: 1) Do keyboard players exhibit a more symmetrical cortical motor hand representation area as indexed by the ILPG than right-handed controls? 2) If musicians indeed differ from controls, are these differences correlated with the age of commencement of musical and motor training?

\section{MATERIALS AND METHODS}

\section{Subjects}

The study was performed on male, right-handed professional musicians. They were recruited from three music conservatories as well as through personal contacts. All of them were actively performing, classically trained musicians who had either finished their 
education or were still students at a music school. No amateur musicians were included. All musicians were keyboard players, with 9 of them playing string instruments in addition. These professional male musicians $(\mathrm{n}=21$; mean age $=26.9$ years, $\mathrm{SD}=4.6)$ were compared to an age- and handedness-matched male control group $(n=30$; mean age $=26.4$ years, $S D=3.9)$. The control subjects in this study were either nonmusicians who had never played a musical instrument or controls who had played an instrument for less than 1 year after age 10 (the latter group comprised about $1 / 3$ of our subjects). We will refer to this group as the nonmusician control group. The control group did not include professional typists, although some used typewriters and computer keyboards for personal communication and correspondence.

In order to exclude possible gender influences, only male subjects were examined in this study. All subjects gave informed consent according to institutional guidelines. Musicians as well as their controls did not have any history of neurologic or psychiatric disease, and all subjects had an unremarkable general physical and neurological examination. Hand preference was assessed with the 12-item Annett questionnaire [Annett, 1970]. Handedness classification was performed by using criteria suggested by previous studies [Witelson and Kigar, 1992; Steinmetz et al., 1992; Jäncke, 1996; Jäncke et al., 1997]. Consistent right-handedness corresponded to performance of all 12 tasks with the right hand, with up to two "either hand" preferences being acceptable. In addition to hand preference, we also assessed distal hand motor skills using an index finger tapping test [Peters and Durding, 1978]. A laterality coefficient was calculated according to $(\mathrm{R}-\mathrm{L}) /$ $(\mathrm{R}+\mathrm{L})$, where L (left) and R (right) were the number of finger taps with either hand within $20 \mathrm{sec}$. Positive values indicated right-handedness, negative values left-handedness. All subjects (musicians and controls) had a laterality coefficient greater than zero and were right-handers according to Annett [1970].

\section{MR imaging}

In vivo MR imaging was performed as previously described and validated [Steinmetz et al., 1990] using a Siemens $1.5 \mathrm{~T}$ magnet (Erlangen, Germany) and a T1-weighted volumetric MR sequence [Frahm et al., 1986] covering the entire brain. Parts of the image not corresponding to telencephalic gray or white matter were removed by an interactively controlled segmentation procedure. Total brain volumes were determined by summing up all voxels $(1.00 \times 1.00 \times 1.17 \mathrm{~mm}$ voxel size) corresponding to telencephalic gray or white matter, but excluding voxels that would correspond to cerebrospinal fluid.

The MR volumes were spatially oriented and normalized in the computerized Human Brain Atlas, whereby only linear transformations were applied (for details concerning the Human Brain Atlas, see Roland and Zilles [1994]). Normalization was performed in order to exclude the influence of intersubject variability in absolute brain size when determining intrasulcal lengths. All brains were spatially oriented according to the coordinate system of Talairach and Tournoux [1988], which enabled us to perform the analyses on horizontal sections which were parallel to the plane determined by the anterior and posterior commissures (AC-PC plane). The presumed representation of the cortical motor hand region was based on previous data from positron emission tomography [Colebatch et al., 1991; Grafton et al., 1991; Kawashima et al., 1994; Schlaug et al., 1994] and functional MRI studies [Kim et al., 1993; Sanes et al., 1995; Yousry et al., 1995; Schlaug et al., 1996]. In some of the latter reports, signal changes in the primary motor cortex in response to single digit movements were found to span up to 40 $\mathrm{mm}$ in the dorso-ventral direction. Even more important, some of these reports specifically stated that signal changes in response to finger movement were observed in the most superior horizontal slices, or up to $\mathrm{z}=69$ when Talairach coordinates were given. These findings are very important for our study, since they served as a justification to perform measurements of the ILPG from Talairach coordinate $\mathrm{z}=69$ to $\mathrm{z}=35$.

Before performing any analyses, we coded all MR data sets. In a random manner, half of the MR data sets were mirrored in the horizontal plane, so that neither subject-identification nor side-of-hemisphere were known to the examiner during the measurements. This ensured optimal blinded conditions. The ILPG was measured in a dorso-ventral sequence of horizontal sections (=region $\mathrm{A}$ ) as the length of the contour line of the posterior bank of the precentral gyrus from the deepest point of the sulcus to a lateral surface tangent which connected the crests of the pre- and postcentral gyrus (Fig. 1). Region B in Figure 1 was arbitrarily defined as the dorsal part of region $A$.

In each horizontal section, the total intrasulcal length as well as an asymmetry score was evaluated (leftright differences in ILPG). An intraobserver reliability [Bartko and Carpenter, 1976] was calculated using a randomly selected subset of 15 brains. Intra- and intersubject reliabilities ranged from $R=0.87$ (for region $\mathrm{A}$ ) to $\mathrm{R}=0.95$ (for region $\mathrm{B}$; both $P<0.0001$ ). Parametric tests (t-tests and analyses of variances) were used for the statistical analysis. Normality was 


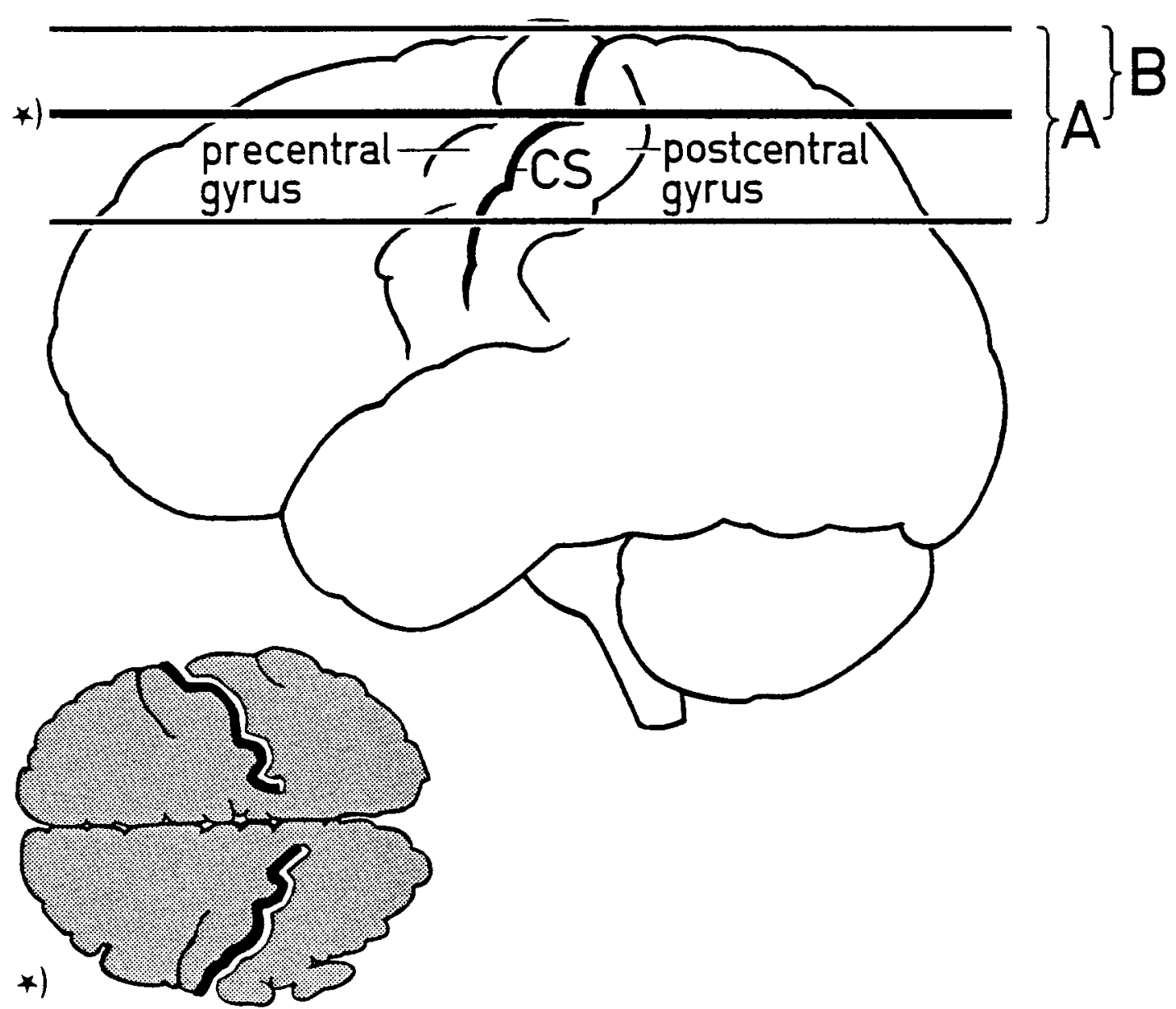

Figure 1.

Schematic lateral view of a human brain (right-handed control). The intrasulcal length of the posterior bank of the precentral gyrus ILPG was analyzed in 35 consecutive horizontal sections (= region $A$; Talairach coordinates $z=69-35)$. *ILPG of both hemispheres is shown as a thick black line on a model horizontal section with

tested using the Kolmogorov-Smirnov test with Lilliefors' correction. Homogeneity of variances was confirmed by a Levene median test (SigmaStat, Jandel, Erkrath, Germany). If these prerequisites for conducting parametric tests were not passed, nonparametric tests were used (Mann-Whitney rank sum test).

Since the tapping scores of the group of musicians were extremely homogeneous, we did not perform intraindividual correlation analysis between tapping scores and ILPG. A Spearman rank order correlation was performed between ILPG size and age of commencement of musical training.

\section{RESULTS}

Preliminary statistical analysis and data inspection did not reveal any significant differences in the anatomy
Talair ach co ordinate $z=54$. CS $=$ central sulcus. The do rsal part of region $A$ ( $=$ region $B$; Talairach coordinates $z=69-54$ ) showed the most pronounced differences between musicians and righthanded controls with respect to asymmetry in ILPG.

of the cortical motor hand representation area between musicians being predominantly keyboard players and musicians playing both keyboard and string instruments. Therefore, these two subgroups were lumped together to constitute the musician group. In order to strengthen the statistical analysis, two mean asymmetry scores were computed, one comprising the entire set of horizontal sections (region A, Talairach coordinates $\mathrm{z}=69-35)$ and one comprising only a dorsal subset (region $B$, Talairach coordinates $\mathrm{z}=69-54$ ). For the asymmetry scores of regions $A$ and $B$, significant leftward asymmetries were evident, both for musicians and controls (Table I). t-tests for independent samples with mean asymmetry scores as dependent variables revealed significant larger leftward asymmetries of both regions for the right-handed 
TABLE I. Left-right differences in ILPG*

\begin{tabular}{lcc}
\hline & $\begin{array}{c}\text { Region A } \\
(\mathrm{z}=69-35)\end{array}$ & $\begin{array}{c}\text { Region } \mathrm{B} \\
(\mathrm{z}=69-54)\end{array}$ \\
\hline RH Musicians $(\mathrm{n}=21)$ & $0.803 \pm 0.419$ & $1.41 \pm 0.586$ \\
RH controls $(\mathrm{n}=30)$ & $1.911 \pm 0.387$ & $3.62 \pm 0.754$
\end{tabular}

* Values are means of all horizontal sections for a region (in $\mathrm{mm}$ ) \pm SEM. Mean differences in ILPG of right-handed (-RH) musicians were significantly lower than those of $\mathrm{RH}$ controls for region $\mathrm{A}$ as well as for B (t-test, $P<0.05)$.

control group compared with the musician group (region $\mathrm{A}, \mathrm{t}(50)=2.08, P<0.05$; region $\mathrm{B}, \mathrm{t}(50)=2.20$, $P<0.05)$.

As depicted in Table I and Figure 2, leftward asymmetry was most pronounced in region $\mathrm{B}$. We therefore analyzed the absolute left and right ILPG of this region in more detail (Fig. 3). These measurements were subjected to a two-way analysis of variance (ANOVA) (classification factor: musicians vs. controls; repeated measurements factor: right vs. left hemisphere). The test revealed a significant interaction $(F=5.02$, $P<0.05)$ as well as main effects $(\mathrm{F}=4.32, P<0.05$ for classification factor; $F=28.5, P<0.0001$ for repeated measurements factor). Subsequent pairwise multiple tests (Student-Newman-Keuls test, $P<0.05$ ) showed a significant greater right intrasulcal length in musicians than in controls, while there was no significant betweengroup difference in this measure for the left hemisphere. Thus, greater symmetry of ILPG in musicians was mainly due to greater ILPG in the hemisphere controlling the nondominant left hand. This result cannot be explained by a simple scaling effect, since absolute brain volumes did not show a significant difference: $1,500 \mathrm{~cm}^{3}(\mathrm{SD}=89)$ for musicians vs. 1,517 $\mathrm{cm}^{3}(\mathrm{SD}=129)$ for controls $(P>0.05)$.

In order to examine whether ILPG was related to the age of commencement of musical training, correlation analyses were performed. We found strong correlations between the time at which musical training had begun and right and left ILPG (Spearman rank order correlation, rs; right hemisphere, $\mathrm{rs}=-0.63$; left hemisphere, $\mathrm{rs}=-0.60$; both $P<0.01$ ). The relationship between early commencement of musical training and right and left ILPG are presented in Figure $4 \mathrm{a}, \mathrm{b}$. No correlation, however, was found with the biological age of the musicians at the time of the study, and with the duration of practice (in years), i.e., the time between onset of musical training and biological age $(P>0.05)$.
Less anatomical asymmetry, combined with greater ILPG, was paralleled by more symmetrical and superior distal finger performance (tapping) in musicians compared with controls. This was confirmed by a two-way ANOVA and subsequent Student-NewmanKeuls tests revealing significant main effects and a significant interaction (classification factor, musicians vs. right-handed controls; repeated measurements factor, right vs. left hand; all $P$ at least $<0.05$; Table II), although in both groups, hand skill asymmetry coefficients significantly differed from zero, indicating a rightward asymmetry in hand performance for both groups (Mann-Whitney rank sum test $\mathrm{T}=790$ for controls, $\mathrm{T}=595.5$ for musicians, both $P<0.0001$ ). The reduced hand skill asymmetry in musicians was due to superior performance of the nondominant hand. This finding parallels the reduced anatomical asymmetry of the ILPG, which was mainly due to a greater ILPG of the nondominant right hemisphere in our right-handed sample.

\section{DISCUSSION}

In this study, we provided evidence for a macrostructural difference in a measure of primary motor cortex size (ILPG) comparing a group of right-handed, professional musicians (predominantly keyboard players) to right-handed nonmusician controls. The selected region (ILPG) is a gross-anatomical correlate of the primary motor cortex [White et al., 1994; Foundas et al., 1995; Amunts et al., 1996] and is also a correlate of the cortical motor hand representation [Grafton et al., 1991; Kim et al., 1993; Sanes et al., 1995; Schlaug et al., 1996]. Musicians had a lesser interhemispheric asymmetry in the ILPG than controls, although musicians still demonstrated a leftward asymmetry in this measure. Reduced ILPG asymmetry in our right-handed musicians was due to a more pronounced enlargement of the ILPG on the right, nondominant hemisphere. Decreased anatomical asymmetry was associated with a reduced asymmetry in distal hand/finger motor skills as assessed by index finger-tapping rates. Furthermore, the reduced hand motor skill asymmetry in musicians was mainly caused by a more pronounced proficiency of the left, nondominant hand, which in turn is controlled by the right hemisphere. In addition to providing evidence for a macrostructural cortical difference comparing musicians to nonmusicians, our results also corroborate a previously found association between the interhemispheric asymmetry of the ILPG and handedness [Amunts et al., 1996]. 


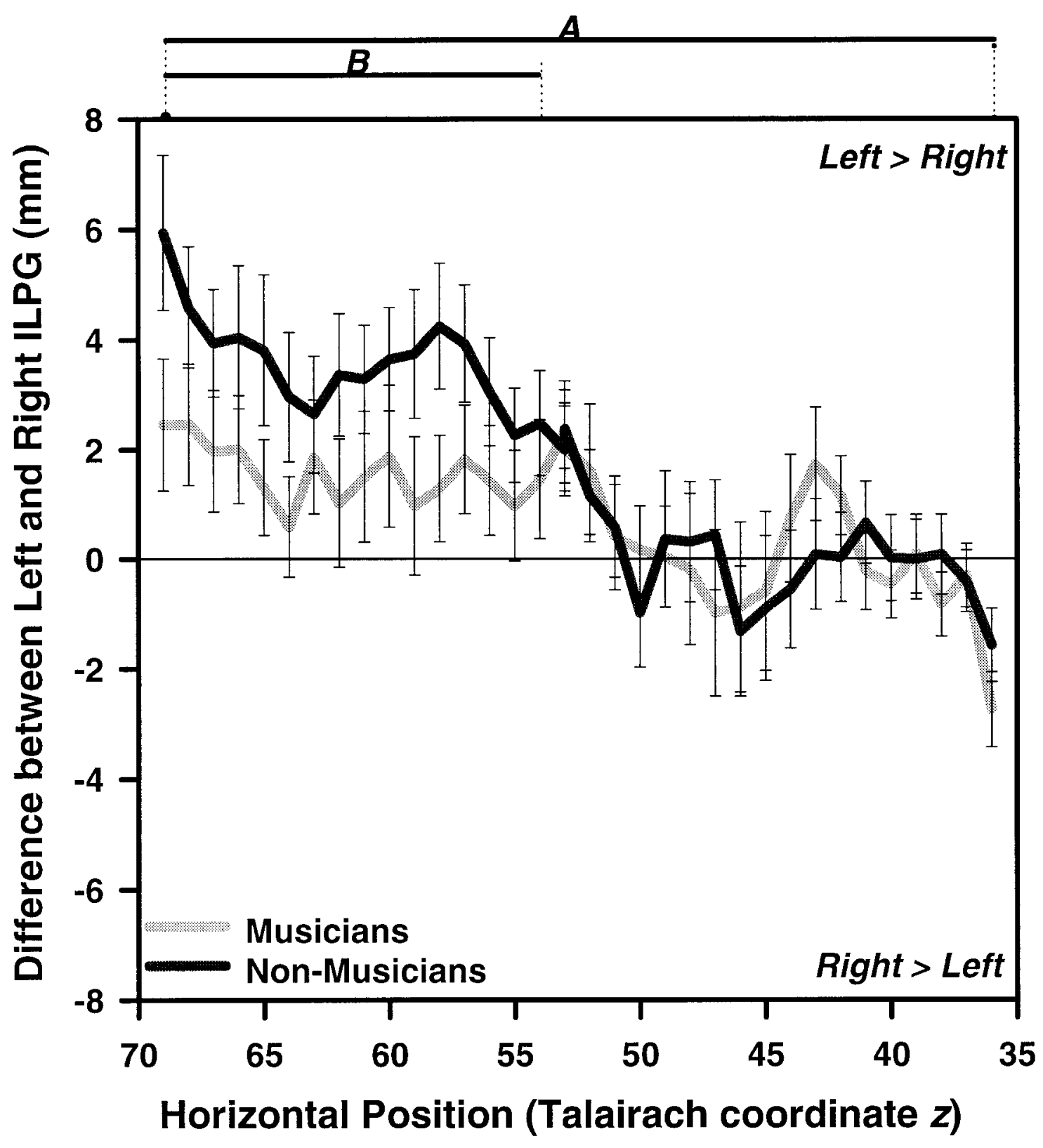

dorsal

ventral

Figure 2.

Differences between left and right ILPG (mean \pm SEM) in 30 right-handed $(-\mathrm{RH})$ controls and $21 \mathrm{RH}$ musicians. Musicians showed significantly lower degrees of asymmetry than controls $(P<0.05)$ in region $A$ of Figure 1 . The most pronounced differ-

ences between musicians and controls were found between Talairach coordinates $z=69-54$ (region $B$ in Fig. 1). Abscissa: plane of sectioning from the most dorsal (Talairach coordinate $z=69$ ) to the most ventral (Talairach coordinate $z=35$ ) position.

Can consistent and in most cases daily practice of complicated bimanual finger sequences lead to detectable, gross-anatomical changes in the motor cortex organization? Or is the predominance of individuals

with a more symmetrical anatomical organization in the hand representation area among our musicians the outcome of a selection process preferring individuals with inborn larger motor cortices and less interhemi- 


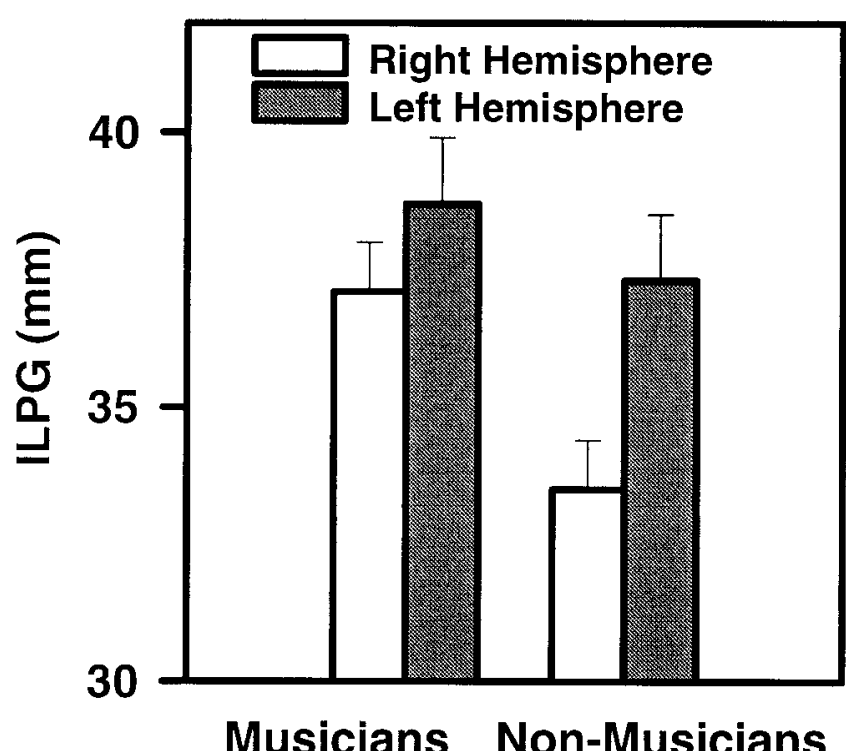

Figure 3.

ILPG (means of all sections of region $B \pm$ SEM) in musicians and non-musicians. Region B: see Figures 1 and 2. A left-larger-thanright asymmetry was found in both groups, but musicians were more symmetrical and showed a significantly greater ILPG on the right hemisphere compared to the controls $(P<0.05)$.

spheric asymmetry? These individuals with larger motor cortices could excel in motor performance during the long period of musical training and therefore surpass competitors "suffering" a more asymmetrical or smaller motor cortex, or both. Our finding of a correlation between ILPG and age of commencement of musical training supports more our initial hypothesis of a training-induced anatomical plasticity. The earlier our musicians began with their bimanual training, the larger were their ILPGs, especially in the hemisphere controlling the nondominant hand. This result is in accordance with current hypotheses that plasticity decreases with aging and that the most pronounced plasticity effects occur during the first years of life [Hallett, 1995]. Considering that the maturation of fiber tracts and cortical neuropil, two determinants of gyral shape [Welker, 1990], are still progressing at age 7 [Yakovlev and Lecours, 1967; Amunts et al., 1995; Armstrong et al., 1995], local structural changes can be assumed to occur in the young brain in response to long-term stimulation.

However, since only about $40 \%$ of ILPG variability is determined by the age of commencement of musical training, the role of other putative determinants for ILPG differences in musicians cannot be completely excluded. We have to admit that we cannot rule out a selectional hypothesis to explain our results. A selec-
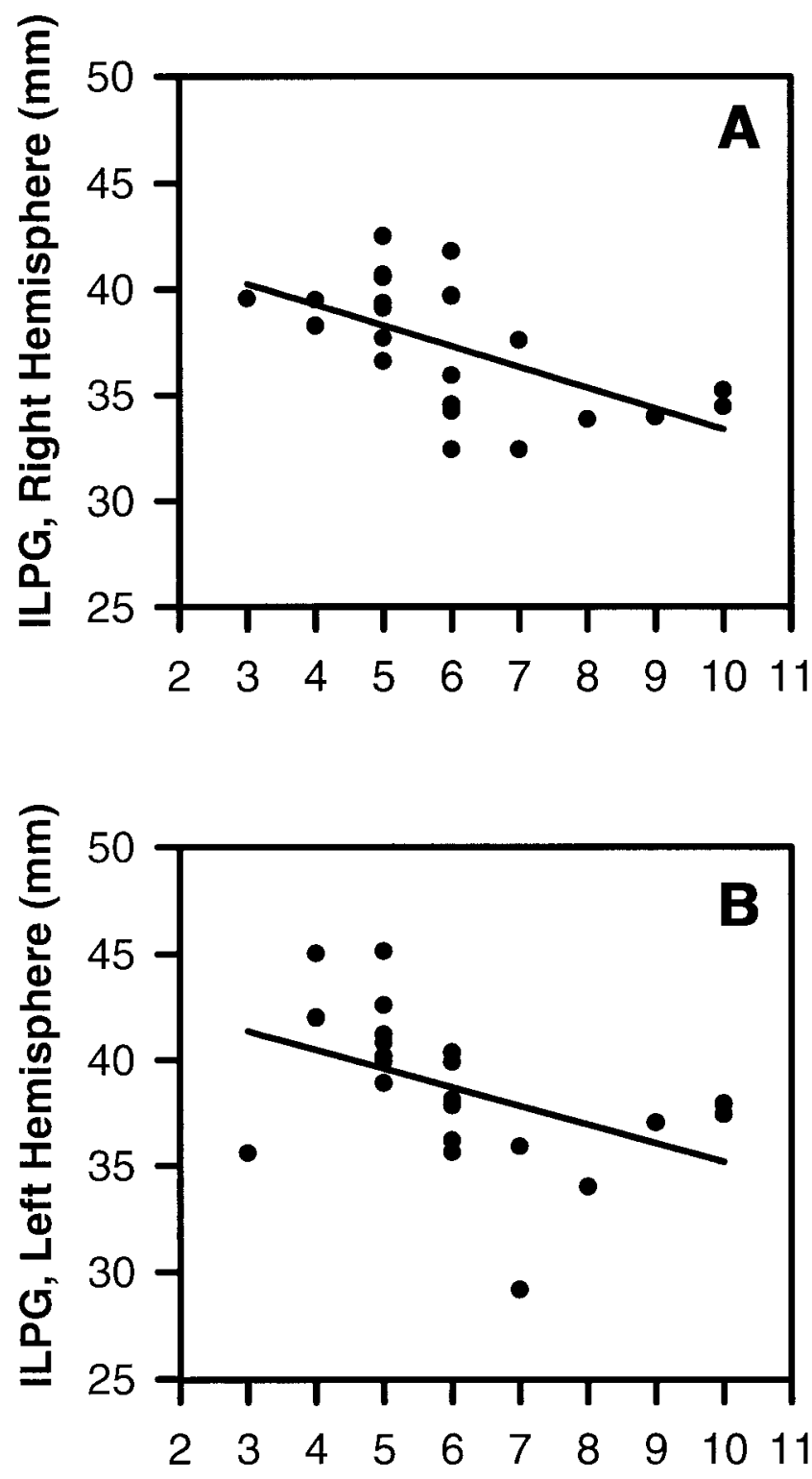

\section{Age of Commencement (years)}

Figure 4.

A: Relationship between age of commencement of musical training and ILPG in the right hemisphere of region B. ILPG was significantly correlated with age of commencement of musical training $(R=-0.63 ; P<0.01)$. B: A similar correlation was found in the left hemisphere $(R=-0.60 ; P<0.01)$.

tional hypothesis would mean that an individual becomes a skilled musician because his motor cortex is larger than average or that an individual establishes a hand dominance because one sensorimotor region is larger than the other. 
TABLE II. Results of index finger tapping test*

\begin{tabular}{lrccc}
\hline & Left hand & Right hand & Mean & $\begin{array}{c}\text { Laterality } \\
\text { coefficients }\end{array}$ \\
\hline RH Musicians $(\mathrm{n}=21)$ & $118.9 \pm 2.44$ & $128.1 \pm 2.44$ & $123.5 \pm 2.31$ & $0.038 \pm 0.007$ \\
RH controls $(\mathrm{n}=30)$ & $96.3 \pm 2.44$ & $111.6 \pm 2.44$ & $103.9 \pm 2.31$ & $0.072 \pm 0.005$
\end{tabular}

* Values are means \pm SEM. The mean tapping scores of right-handed (-RH) musicians were significantly higher than those of RH controls; left-hand scores were more different than right-hand scores (two-way ANOVA; $P<0.01$ ). Laterality coefficients indicated significantly decreased asymmetry in musicians compared to controls $(P<0.05)$

After all, complicated bimanual finger movements are the result of the interaction of several motor circuits, including premotor and supplementary motor cortex, basal ganglia, and the cerebellum. Thus, these circuits could modify the effect of hand skill training on the motor cortex and cause variability in ILPG. The cerebellum, for instance, was shown to play a special role in learning the timing of movement [Raymond et al., 1996], whereby regulation of movements seems to be performed independently in each half of the cerebellum for the ipsilateral hand [Franz et al., 1996].

The finding of a structural difference between the motor cortices of musicians and nonmusicians supplements existing data on long-term functional adaptation in the human cerebral cortex, e.g., changes in the cortical representation of body parts [Cohen et al., 1991; Ramachandran et al., 1992; Flor et al., 1995; Mano et al., 1995; Pascual-Leone et al., 1995b; Elbert et al., 1995]. Flor et al. [1995] and Elbert et al. [1995] revealed differences in the functional organization of somatosensory cortical areas that would suggest long-term adaptation. Long-term representational changes in the motor cortex as a function of practice were shown by Pascual-Leone et al. [1995a] and by Karni et al. [1995]. Using transcranial magnetic stimulation, it was shown that the motor cortical output map to the reading hand in Braille readers was even modifiable after several hours of training [Pascual-Leone et al., 1995b]. The rapid time course of these changes makes an alteration in the effectiveness of existing synaptic connections and long-term potentiation more likely than actual changes in the cortical microarchitecture [Iriki et al., 1989; Jacobs and Donoghue, 1991; Hess and Donoghue, 1994]. Studies done on patients with upper limb amputation, and patients with ischemic or tumorous brain lesions, provided further evidence that the cortical sensory and motor maps are modifiable and can exhibit long-term adaptations, including spatial relocation or enlargement of cortical maps [Cohen et al., 1991; Ramachandran et al., 1992; Flor et al., 1995; Mano et al., 1995; Seitz et al., 1996]. Similarly, nonhuman studies have shown that intense motor activity can lead to longer-lasting functional and macrostructural cerebral changes: use-dependent alterations of movement representations were found in the primary motor cortex of adult squirrel monkeys at least several days after acquisition of motor skills [Nudo et al., 1996]. In another study, a time-dependent increase in the number of synapses per neuron in the contralateral motor cortex after unilateral sensorimotor lesions was found, which was interpreted as a compensatory change in the hemisphere controlling the nonimpaired forelimb [Jones et al., 1996]. A wealth of data exists to support the contention that cortical plastic changes occur either after peripheral lesions interfering with sensory input or after central lesions interfering with the cortical circuitry [Donoghue and Sanes, 1988; Merzenich et al., 1988; Sanes et al., 1990; Recanzone et al., 1990; Jacobs and Donoghue, 1991; Rauschecker, 1995].

A series of studies [Black et al., 1990; Isaacs et al., 1992; Anderson et al., 1994; Swain et al., 1994] investigated the underlying microarchitecture of gross macrostructural adaptations in the cerebellum by subjecting rats to different motor tasks. One group of rats was forced to perform acrobatic tasks while another group was forced to walk in a treadmill. Both groups were compared to a voluntary exercise group. Acrobatic training resulted in an increase in the number of synapses and a greater volume of glial per Purkinje cells in the cerebellar cortex, while there was an increase in the microvasculature in the forced exercise group. These studies are very important for interpreting our results, since these results would support the hypothesis that intense and prolonged motor activity can lead to microstructural changes (e.g., increase in the number of synapses, glial cells, and capillaries). These volumetric changes in the neuropil compartment can certainly lead to changes detectable by macrostructural methods. Recent work from the same group revealed changes in the thickness of the primary motor cortex [Anderson et al., 1992] and, more specifi- 
cally, an increase in the number of synapses per neuron in the motor cortex [Kleim et al., 1996] of adult rats which were trained on an acrobatic motor task. Taken together, these studies support our interpretation of the structural differences in the motor cortex between keyboard players and controls as a structural compliance in response to an intense and early hand skill training. However, a longitudinal MR study will be needed for a direct demonstration of training-induced morphological plasticity.

\section{ACKNOWLEDGMENTS}

This work was supported by Deutsche Forschungsgemeinschaft grant SFB 194, A6. We thank Kristina Rascher for helpful discussion.

\section{REFERENCES}

Amunts K, Istomin VV, Schleicher A, Zilles K (1995): Postnatal development of the human primary motor cortex: A quantitative cytoarchitectonic study. Anat Embryol (Berl) 192:557-571.

Amunts K, Schlaug G, Schleicher A, Steinmetz H, Dabringhaus A, Roland PE, Zilles K (1996): Asymmetry in the human motor cortex and handedness. Neuroimage 4:216-222.

Anderson BJ, Eckburg PB, Relucio KI, Greenough WT (1992): Thickness of the motor cortex increases following 28 days of exercise or motor skill training. Soc Neurosci Abstr 18:346.

Anderson BJ, Li X, Alcantara AA, Isaacs KR, Black JE, Greenough WT (1994): Glial hypertrophy is associated with synaptogenesis following motor-skill learning, but not with angiogenesis following exercise. Glia 11:73-80.

Annett M (1970): A classification of hand preference by association analysis. Br J Psychol 61:303-321.

Armstrong E, Schleicher A, Omram H, Curtis M, Zilles K (1995): The ontogeny of gyrification. Cereb Cortex 1:56-63.

Black JE, Isaacs KR, Anderson BJ, Alcantara AA, Greenough WT (1990): Learning causes synaptogenesis, whereas motor activity causes angiogenesis, in cerebellar cortex of the rat. Proc Natl Acad Sci USA 87:5568-5572.

Bartko JJ, Carpenter WT (1976): On the methods and theory of reliability. J Nerv Ment Dis 163:307-317.

Christman S (1993): Handedness in musicians: Bimanual constraints on performance. Brain Cogn 22:266-272.

Cohen LG, Bandinelli S, Findeley TW, Hallett M (1991): Motor reorganization after upper limb amputation in man. Brain 114:615627.

Colebatch JG, Deiber M-P, Passingham RE, Friston KJ, Frackowiak RSJ (1991): Regional cerebral blood flow during voluntary arm and hand movements in human subjects. J Neurophysiol 65:13921401.

Donoghue JP, Sanes JN (1988): Organization of adult motor cortex representation patterns following neonatal forelimb nerve injury in rats. J Neurosci 8:3221-3332.

Elbert T, Pantev C, Wienbruch C, Rockstroh B, Taub E (1995): Increased cortical representation of the fingers of the left hand in string players. Science 270:305-307.

Flor H, Elbert T, Knecht S, Wienbruch C, Pantev C, Birbaumer N, Larbig W, Taub E (1995): Phantom-limb as a perceptual correlate of cortical reorganization following arm amputation. Nature 375:482-484.

Foundas AL, Hong K, Leonard CM, Heilman KM (1995): Hand preference and MRI asymmetries of the human central sulcus. Soc Neurosci Abstr 21:438.

Frahm J, Haase A, Matthaei D (1986): Rapid three-dimensional MR imaging using the FLASH technique. J Comput Assist Tomogr 10:363-368.

Franz CA, Ivry RB, Helmuth LL (1996): Reduced timing variability in patients with unilateral cerebellar lesions during bimanual movements. J Cogn Neurosci 8:107-118.

Grafton ST, Woods RP, Maziotta JC, Phelps ME (1991): Somatotopic mapping of the primary motor cortex in humans: Activation studies with cerebral blood flow and positron emission tomography. J Neurophysiol 66:735-743.

Hallett M (1995): The plastic brain. Ann Neurol 38:4-5.

Hess G, Donoghue JP (1994): Long-term potentiation of horizontal connections provides a mechanism to reorganize cortical motor maps. J Neurophysiol 71:2543-2547.

Iriki A, Pavlides C, Keller A, Asanuma H (1989): Long-term potentiation of motor cortex. Science 245:1385-1387.

Isaacs KR, Anderson BJ, Alcantara AA, Black JE, Greenough WT (1992): Exercise and the brain: Angiogenesis in the adult rat cerebellum after vigorous physical activity and motor skill learning. J Cereb Blood Flow Metab 12:110-119.

Jacobs KM, Donoghue JP (1991): Reshaping the cortical motor map by unmasking latent intracortical connections. Science 251:944947.

Jäncke L (1996): The hand performance test with a modified time limit instruction enables the examination of hand performance asymmetries in adults. Percept Mot Skills 82:735-738.

Jäncke L, Schlaug G, Steinmetz H (1997): Hand skill asymmetry in professional musicians. Brain Cogn, in press.

Jenkins IH, Brooks DJ, Nixon PD, Frackowiak RS, Passingham RE (1994): Functional reorganization of primary somatosensory cortex in adult owl monkeys after behaviorally controlled tactile stimulation. J Neurosci 14:3775-3790.

Jones TA, Kleim JA, Greenough WT (1996): Synaptogenesis and dendritic growth in the cortex opposite unilateral sensorimotor cortex damage in adult rats-A quantitative electron microscopic examination. Brain Res 733:142-148.

Karni A, Meyer G, Jezzard P, Adams MM, Turner R, Ungerleider LG (1995): Functional MRI evidence for adult motor cortex plasticity during motor skill learning. Nature 377:155-158.

Kawashima R, Roland PE, O'Sullivan BT (1994): Regional cerebral blood flow changes of cortical motor areas and prefrontal areas in humans related to ipsilateral and contralateral hand movement. J Neurosci 14:3462-3474.

Kim SG, Ashe J, Hendrich K, Ellermann J, Merkle H, Ugurbil K, Georgopoulos AP (1993): Functional magnetic resonance imaging of motor cortex: Hemispheric asymmetry and handedness. Science 261:615-617.

Kleim JA, Lussnig E, Schwarz ER, Comery TA, Greenough WT (1996): Synaptogenesis and FOS expression in the motor cortex of the adult rat after motor skill learning. J Neurosci 16:4529-4535.

Mano Y, Nakamuro T, Tamura R, Takayanagi T, Kawanishi K, Tamai S, Mayer RF (1995): Central motor reorganization after anastomosis of the musculucutaneous and intercostal nerves following cervical root lesion. Ann Neurol 38:15-20.

Merzenich MM, Recanzone G, Jenkins WM, Allard TT, Nudo RJ (1988): Cortical representational plasticity. In: Rakic P, Singer W (eds): Neurobiology of Neocortex. New York: John Wiley \& Sons, pp 41-47. 
Nudo RJ, Jenkins WM, Merzenich MM, Prejean T, Grenda R (1992): Neurophysiological correlates of hand preference in primary motor cortex of adult squirrel monkeys. J Neurosci 12:2918-2947.

Nudo RJ, Milliken GW, Jenkins WM, Merzenich MM (1996): Usedependent alterations of movement representations in primary motor cortex of adult squirrel monkeys. J Neurosci 16:785-807.

Pascual-Leone A, Cohen LG, Dang N, Brasil-Neto JP, Cammarota A, Hallet M (1993): Acquisition of fine motor skills in humans is associated with the modulation of cortical motor output maps. Neurology 43:157.

Pascual-Leone A, Dang N, Cohen LG, Brasil-Neto J, Cammarota A, Hallett M (1995a): Modulation of muscle responses evoked by transcranial magnetic stimulation during the acquisition of new fine motor skills. J Neurophysiol 74:1037-1045.

Pascual-Leone A, Wassermann EM, Sadato N, Hallett M (1995b): The role of reading activity on the modulation of motor cortical outputs to the reading hand in Braille readers. Ann Neurol 38:910-915.

Peters M, Durding BM (1978): Handedness measured by finger tapping: A continuous variable. Can J Psychol 32:257-261.

Ramachandran VS, Stewart M, Rogers-Ramachandran DS (1992): Perceptual correlates of massive cortical reorganization. Neuroreport 3:583-586.

Rauschecker JP (1995): Compensatory plasticity and sensory substitution in the cerebral cortex. Trends Neurosci 18:36-43.

Raymond JL, Lisberger SG, Mauk MD (1996): The cerebellum: A neuronal learning machine? Science 272:1126-1131.

Recanzone GH, Allard TT, Jenkins WM, Merzenich MM (1990): Receptive-field changes induced by peripheral nerve stimulation in S1 of adult cats. J Neurophysiol 63:1213-1225.

Roland PE, Zilles K (1994): Brain atlases-A new research tool. Trends Neurosci 17:458-467.

Sanes JN, Suner S, Donoghue JP (1990): Dynamic organization of primary motor cortex output to target muscles in adult rats. I. Long-term patterns of reorganization following motor or mixed peripheral nerve lesions. Exp Brain Res 79:479-491.

Sanes JN, Donoghue JP, Thangaraj V, Edelman RR, Warach S (1995): Shared neural substrates controlling hand movements in human motor cortex. Science 268:1775-1777.

Schlaug G, Knorr U, Seitz R (1994): Inter-subject variability of cerebral activations in acquiring a motor skill: a study with positron emission tomography. Exp Brain Res 98:523-534.

Schlaug G, Jäncke L, Huang Y, Staiger JF, Steinmetz H (1995a): Increased corpus callosum size in musicians. Neuropsychologia 33:1047-1054
Schlaug G, Jäncke L, Huang Y, Steinmetz H (1995b): In vivo evidence of structural brain asymmetry in musicians. Science 267:699-701.

Schlaug G, Sanes JN, Thangaraj V, Darby DG, Jäncke L, Edelman RR, Warach S (1996): Cerebral activation covaries with movement rate. Neuroreport 7:879-883.

Seitz RJ, Huang Y, Knorr U, Tellmann L, Herzog H, Freund HJ (1996): Large-scale plasticity of the human motor cortex. Neuroreport $6: 742-744$.

Steinmetz H, Rademacher J, Jäncke L, Huang Y, Thron A, Zilles K (1990): Total surface of temporoparietal intrasylvian cortex: Diverging left-right asymmetries. Brain Lang 39:357-372.

Steinmetz H, Jäncke L, Kleinschmidt A, Schlaug G, Volkman J, Huang Y (1992): Sex, but no hand difference in the isthmus of the corpus callosum. Neurology 47:749-752.

Swain RA, Harris AB, Wiener EC, Morris HD, Swain CR, Lauterbur PC, Greenough WT (1994): fMRI of rat motor cortex following physical exercise. Soc Neurosci Abstr 20:147.

Talairach J, Tournoux P (1988): Coplanar Stereotaxic Atlas of the Human Brain, Stuttgart: Thieme.

Waldron EM, Anton BS (1995): Effects of exercise on dexterity. Percept Mot Skills 80:883-889.

Wang X, Merzenich MM, Sameshima K, Jenkins WM (1995): Remodeling of hand representation in adult cortex determined by timing of tactile stimulation. Nature 378:71-75.

Welker W (1990): Why does cerebral cortex fissure and fold? A review of determinants of gyri and sulci. In: Jones EG, Peters A (eds): Cerebral Cortex, Vol 8B. New York: Plenum Press, pp 3-136.

White LE, Lucas G, Richards A, Purves D (1994): Cerebral asymmetry and handedness. Nature 368:197-198.

Witelson SF, Kigar DL (1992): Sylvian fissure morphology and asymmetry in men and women: Bilateral differences in relation to handedness in men. J Comp Neurol 323:326-340.

Yakovlev PI, Lecours A-R (1967): The myelogenetic cycles of regional maturation of the brain. In: Minkowski A (ed): Regional Development of the Brain in Early Life. Oxford: Blackwell, pp 3-70.

Yousry TA, Schmid UD, Jassoy AG, Schmidt D, Eisner WE, Reulen H-J, Reisner MF, Lissner J (1995): Topography of the cortical motor hand area: Prospective study with functional MR imaging and direct motor mapping at surgery. Neuroradiology 195:23-29. 